\title{
PERCEPTIONS OF PHYSICAL ACTIVITY, ACTIVITY PREFERENCES AND HEALTH AMONG A GROUP OF ADULT WOMEN IN URBAN GHANA: A PILOT STUDY
}

\author{
Y. A. TUAKLI-WOSORNU ${ }^{1}$, M. ROWAN ${ }^{1}$ and J. GITTELSOHN ${ }^{2}$ \\ ${ }^{1}$ Department of Physiatrist, Hospital for Special Surgery, New York, USA; Department of International \\ Health and the Centre for Human Nutrition, ${ }^{2}$ Department of International Health, Johns Hopkins Bloomberg \\ School of Public Health, Baltimore, USA
}

DOI: http://dx.doi.org/10.4314/gmj.v48i1.1

Corresponding Author: Dr. Yetsa .A. Tuakli-Wosornu

Email: yetsa.kehinde@gmail.com

Conflict of Interest: None declared

\section{SUMMARY}

Background: Obesity and other lifestyle-related chronic diseases impact urban West African women at high rates. Physical activity (PA) can improve these health outcomes, but there is little published data on the associated psychosocial predictors in this population.

Objectives: We aimed to explore preliminary associations between perceptions of PA, PA behaviours, and health in a group of Ghanaian women.

Methods: Non-experimental, cross-sectional case study using a mixed-methods approach. Focus groups and in-depth interviews with a convenience sample of Ghanaian women, fitness trainers and clergy comprised the qualitative phase. A self-administered survey $(n=218)$ comprised the quantitative phase. Constant comparative method, logistic regression, component and factor analyses were used for analysis.

Results: Women viewed activities of daily living like housework as PA; rarely utilized organized fitness facilities; understood "rigorous" PA as professional male athleticism; and took interest in socialized PA. Mean age was 49.4 years. Mean body mass index was 30.3 $\mathrm{kg} / \mathrm{m} 2$. The majority $(75.9 \%)$ reported exercising sometimes or often. Half (48.4\%) reported a lifestylerelated chronic disease. "Weight loss," "health concerns" and "increased energy," were top motivators for PA. "Can't find the time," "work/family obligations," and "don't have a facility" were top barriers. Presence of hypertension, hypercholesterolemia and prior weight loss correlated with the slimming motivator (adjusted odds ratio 2.59, $\mathrm{p}=0.008$; AOR 3.56, $\mathrm{p}=0.012$; AOR $3.36, \mathrm{p}=<0.001$ ).

Conclusion: Among those surveyed, PA motivators and barriers were associated with demographics, PA exposure, and health status. Further research on unique PA perceptions, behaviours and health could catalyze health promotion through culturally relevant fitness programming.
Keywords: physical activity, Ghana, women, facilitators, barriers.

\section{INTRODUCTION}

Regular physical activity (PA) and proper nutrition are the two major determinants of a healthy lifestyle. Taken individually and collectively, they can help reduce obesity-related risk of chronic disease, and improve indicators related to chronic disease (e.g., blood lipid and glucose levels). ${ }^{1}$ The socio-cultural climate of urban West Africa, increasingly defined by technological advances in transportation and food, enables a sedentary lifestyle and related diseases. ${ }^{2,3,4,5}$

The regional prevalence of obesity is approximately $10 \%$ and rising: trend analyses demonstrate obesity prevalence has more than doubled in the past decade and a half. ${ }^{6}$ Women (versus men) and urban dwellers (versus rural) have the highest rates of obesity. ${ }^{6}$ The regional prevalence of physical inactivity is $13 \%$. Women (versus men), older adults (> age 50) and urban dwellers (versus rural) have the highest rates of inactivity. 6 In Ghana, the prevalence of obesity is between $10.7 \%$ and $14.1 \%$. Women are disproportionately affected.

Obesity and overweight are increased among married, non-smoking and employed Ghanaian adults. ${ }^{7,8}$ Of the 1.6 million residents in Greater Accra (capital city), $16.1 \%$ are obese. 8,9 These are the highest rates of obesity in Ghana. No PA or inactivity data is available for women living in Accra.

Studies indicate that culture is a key determinant of behaviors associated with overweight and obesity. Specific cultural beliefs about PA, which often arise from unique ethnic identities and exposures, play a strong role in women's decision to adopt and maintain regular 
PA. ${ }^{10,11}$ For instance, low-income urban AfricanAmerican women may view PA as unfeminine and in conflict with the perceived attractiveness of excess body fat. ${ }^{12}$ Among this population, perceived barriers also include the lack of available and/or safe exercise facilities. ${ }^{12}$ Similarly, perceived time-consumption and lack of facilities may limit PA among older, multiethnic American adults, but socialized PA and group activities motivate this group. ${ }^{13}$ Primary PA barriers and facilitators among Filipino, Native American, Korean and African adults in the U.S include health risks such as developing or preventing high blood pressure and diabetes mellitus. ${ }^{13,14}$

In South Africa, many rural women may be unconcerned about obesity and its associated disease risks. ${ }^{15,16}$ There may also be negative social pressure to lose weight among this group. ${ }^{15,16}$ Culturally specific perceptions have important implications for the design and success of PA programs for ethnically diverse women. Although information on West African women's perceptions of body weight is increasing, data on PA behaviors, motivators and barriers is threadbare.

Ghana has produced one sub-national population-based study that included an assessment of PA. ${ }^{4}$ Despite data indicating that urban women have the highest rates of lifestyle-related disease in Ghana, ${ }^{17}$ literatures on the psychosocial precursors to associated health behaviors is absent. This literature gap is the driving force behind our investigation. The associations between culture and health perceptions, ${ }^{10}$ and increased PA and reduced disease risk are clear. A better understanding of Ghanaian women's perceptions of PA and health can help direct effective, culturally-relevant PA interventions. Given the high prevalence of inactivity (13\%) and chronic disease in the population, such programs are becoming increasingly important.

\section{Specific aims}

The specific aims of this study were to: 1) collect and summarize demographic and obesity-related health data on a cohort of women aged forty and above in Accra, Ghana, 2) conduct qualitative and quantitative analyses of social, cultural and individual psychosocial determinants of PA among the cohort, and 3) use these data to demonstrate the need for large-scale formative research which may be used to design a cohort-specific fitness intervention. The conceptual framework incorporated individual factors such as PA behaviors and disease status, and environmental factors such as social support and family dynamics; two key determinants of PA in multi-ethnic adults. ${ }^{18}$

\section{METHODS}

Study design
This was a descriptive, cross-sectional analysis. The mixed-methods paradigm was used to increase the comprehensiveness of data collection. The data collector was a graduate student trained by the senior author.

\section{Setting}

Qualitative interviews were carried out in:

1) Individual households,

2) A private section of the church sanctuary,

3) The common room of a local gym, and

4) The pastoral offices of the church. Data collection was primarily conduced in a single, well-attended mega church in a central location within Accra, Ghana.

Site selection was based on the high number of congregants who matched the study's selection criteria and the similarities between the sample and general population in greater Accra (heterogeneity in individual residences and socio-demographic characteristics, but homogeneity in religious affiliation and language). The 2000 Ghanaian national census confirms that of the 1.6 million residents of Accra, $88 \%$ live in an urban setting and $69 \%$ self-identify as Christian. ${ }^{9}$ There are five mega churches in Greater Accra, broadly defined as churches at which total Sunday attendance approaches or exceeds 2000 parishioners. ${ }^{19}$

The study site is the most diverse and well-attended mega church in Greater Accra. Located on the thirdlargest motorway in Accra, the church is easily accessed by public and private transportation as well as church-organized shuttles. Congregants travel up to 4.2 kilometers to reach the site. Though diverse, the congregation is almost exclusively English speaking. All church services are conducted in English.

\section{Participants and recruitment}

Survey subjects were purposively recruited and consented to participate by trained clergy members and the authors. Criteria for inclusion included female gender, English-literacy, age ( $\geq 40$ years), ethnicity (Ghanaian), place of residence (urban Greater Accra), religion (Christianity), and membership at the chosen mega church. Survey subjects were pre-notified via brief verbal church announcements on two consecutive Sundays prior to the study.

On the survey date, the pastor invited all women at or above age forty to participate in the study at the end of two morning church services. Eligibility criteria were verified at recruitment. Of 1035 parishioners who turned in a census slip at the beginning of the service, 671 were ineligible by virtue of gender (male) or age ( $<40$ years). 363 women were eligible for participation in the study, 282 surveys were completed and 218 sur- 
veys were analyzed. Figure 1 illustrate the sampling procedure.

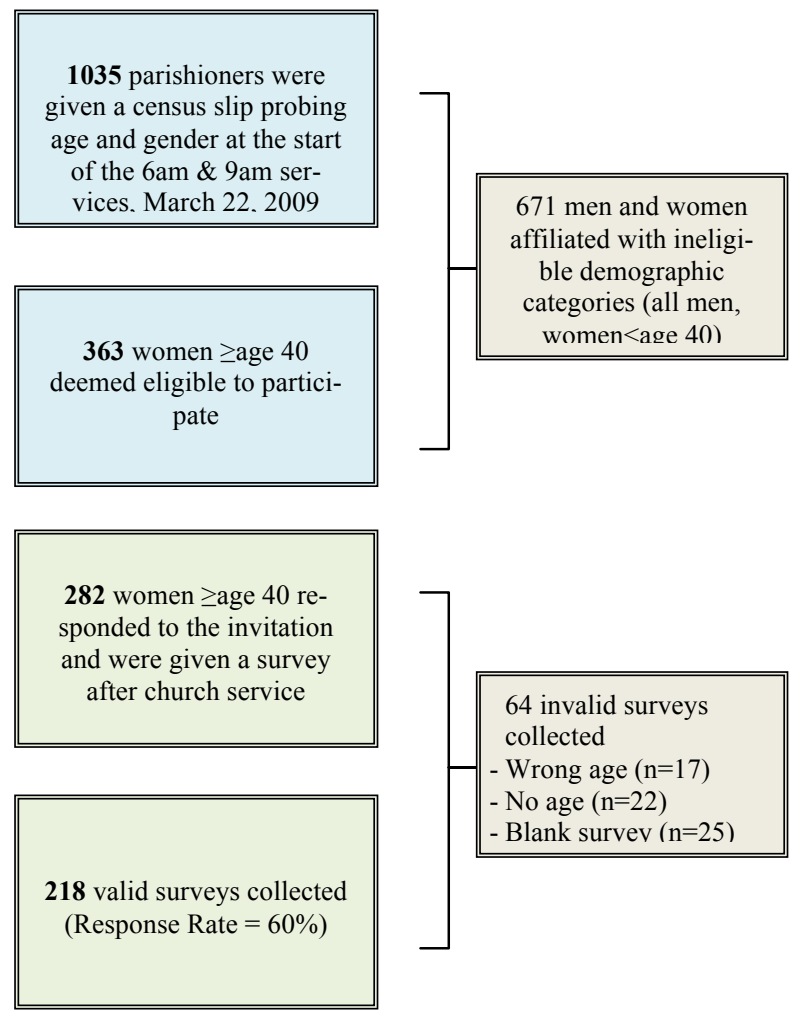

Figure 1 Standard consort diagram of the study illustrating people who were probed for participation in the study, women who were deemed eligible for participation, women who completed surveys and women for whom survey data was deemed valid

\section{Ethical considerations}

Prior to data collection, the Johns Hopkins Bloomberg School of Public Health Institutional Review Board approved this study. Each participant gave oral informed consent after the study rationale, procedure, risks and benefits were described using common language.

\section{Qualitative Data}

A total of 3 focus group discussions and 6 semistructured in-depth interviews were carried out. The three focus groups were: 1) adult Ghanaian women aged $58-71$ years, 2) fitness professionals aged $27-$ 42 years, and 3) clergy aged $40-72$ years. An openended interview guide was used for the individual indepth interviews, which included: 1) three adult Ghanaian women, aged 52, 57 and 58 years, 2) one local fitness professional, aged 42 years, and 3) two local clergy, aged 58 and 60 years. All qualitative data were recorded, transcribed and analyzed using the constant comparative method. ${ }^{20}$ Key themes were identified after initial review of the text.
They were subsequently refined and used to inform the development of a questionnaire.

\section{Quantitative Data}

Quantitative data were derived from a selfadministered 19-item questionnaire, constructed using interview data and common physical activity questions found in the literature. The survey was initially administered to a convenience sample of Ghanaian women $(\mathrm{n}=50$, mean age $50.2 \mathrm{yrs}, 40 \%$ college educated), who were recruited from a mega church in Accra, Ghana (see "mega church" definition under Setting subheading).

Given the high attendance, diversity of congregants and physical centrality of the mega church selected (see setting sub-heading), self-administered survey methodology maximized efficiency. Our collaborating church leader's purposively selected representative participants, who met our selection criteria (see Population sub-heading). 
At the time of recruitment, collaborators informed study participants about the survey purpose and methodology, and were given ample time to ask questions regarding risks, benefits, and expectations.

The surveys were completed and discussed with the initial 50 respondents, research assistants, and the principle investigator. Using this feedback, the instrument was refined for future use. Surveys were individually administered and written. Survey results were tabulated in Microsoft Excel $^{\mathrm{TM}}$ then imported into STATA $^{\mathrm{TM}}$ software for analysis.

\section{Analysis methods}

Qualitative data was organized into key themes. Data from each group of respondents (women, trainers and clergy) were analyzed separately before they were combined into a summative review. Using the constant comparative method, main themes were extracted from the summative review and utilized to develop the survey.

Quantitative data were evaluated in STATA $^{\text {TM }}$ analysis software. Demographic trends in health status, PA history, current PA and perceptions of PA were identified using logistic regression. Multi-variable relationships were identified and tested using component and factor analysis. Once the top three motivators and barriers to PA were identified, they were separately regressed on four covariates: age, educational status (college), marital status (married) and body mass index (BMI).

To better anthropomorphize the results and offer a more lifestyle-oriented rendering of survey data, theoretical respondent profiles were generated from factor analyses. Specifically, respondents were stratified by four groups, determined by the age variable's strength of association with all factors, the strength with which age was associated with other demographic variables, and each motivator and barrier loaded was observed.

The pattern of variable loading was observed and interpolated into respondent profiles that summarize the cohort. Motivators and barriers to PA results were also compared to previously published data among agesimilar, multi-ethnic adults to identify similarities and differences between our subjects and other populations.

\section{RESULTS}

\section{Qualitative results}

\section{Adult women}

Focus group discussions and in-depth interviews revealed three key themes: health (awareness and concerns), low self-efficacy (conscious lack of knowledge compounded with time- and family-obligations to constrain women from engaging in PA), and receptiveness (interest in PA education and media and/or community fitness programming).

Time constraints and personal safety were the most commonly noted barriers. As one participant said, "It takes a lot of time in the day and we don't have that time... I try to do exercises that are safe for me, for my age, but I don't always know what I can and cannot do..." Multiple women mentioned the importance of safety when exercising, reflecting that they desired more knowledge of safe exercises, age-appropriate exercise programs, and healthy eating habits.

"Nutrition is the major factor, I know. I eat healthy and stay away from rice, banku...those heavy starch[es]..." On fitness videos and media programs, a 58-year-old business-owner confided, “...I can't imagine doing that. I'm exhausted watching the [young] girls."

Women were interested in the social elements of fitness, admitting a preference for group-based activities where social interaction was foundational. On exercise preferences, a 62-year-old homemaker said "yes, we need to learn. We play golf. It's safe and very meditative... We meet each other on the course."

\section{Fitness professionals}

Focus group discussions and in-depth interviews among local fitness professionals revealed three key themes: a participation void, culturally unique thinking, and strong interest among adult Ghanaian women. Trainers explained that Ghanaian women may find fitness facilities abstruse and alien, associate rigorous PA with young men, desire privacy and connectedness while exercising, and formulate some perceptions about PA from observing professional athletes.

In the focus group, barriers identified included: 1) "older women don't come to the gym. They don't think it is for them," 2) "if they come in, they get intimidated by weights and machines and they don't feel strong to ask questions," 3) "they see the footballers and they think sports and fitness is for the young men," 4) "They fear that if they cannot sustain a fitness program, they will end up worse than they started... The local footballers are fit while they are playing but when they retire, they become very fat... [Women] fear that, so they don't want to start exercise."

During an in-depth interview, a 42-year-old personal trainer who studied under his father and educates numerous trainers in the greater Accra region stated, "the women of that age, they prefer us trainers to come to their home so they can exercise in a private setting... They fear the gym, so they prefer to walk with friends around their compounds..." Trainers repeatedly noted that women report feeling intimidated by unfamiliar 
fitness machinery and a typically male-dominated gym population.

\section{Clergy}

Focus group and in-depth interviews among clergy uncovered one common theme: an interest in faithbased fitness programming both for personal health reasons and community service. A group of elderly male clergy chorused: "yes, we want to know what to do for our women... At times, after they marry, they give up... But what about the men? We want to know how to be fit, too [laughter]."

In-depth interviews with the pastor and first lady (pastor's wife) revealed an awareness of both the preventive health benefits of fitness and the prevalence of lifestyle-related disease in the community. "Health is one of the top items on our [church] agenda this year... I tell [them] to bring me fresh fruits and vegetables at every meal... But we don't know what is good to do for exercise. And we need it." The pastor's wife continued, "And all the diseases we know are connected to obesity..." She further confessed, "If we as a congregation all do it together, I could get involved to motivate the women..."

\section{Main themes}

The main themes were as follows: health concerns and woes, time constraints, social elements of PA and receptiveness of all parties to learn and improve knowledge of PA.

\section{Quantitative results \\ Demographics}

Of the 218 participants, $96.7 \%$ self-identified as Ghanaian nationals, and $84.9 \%$ reported living in Accra city (Table 1). Mean age was 49.4 years. The majority of women were younger than age 60 .

Table I: Baseline demographics of the study cohort

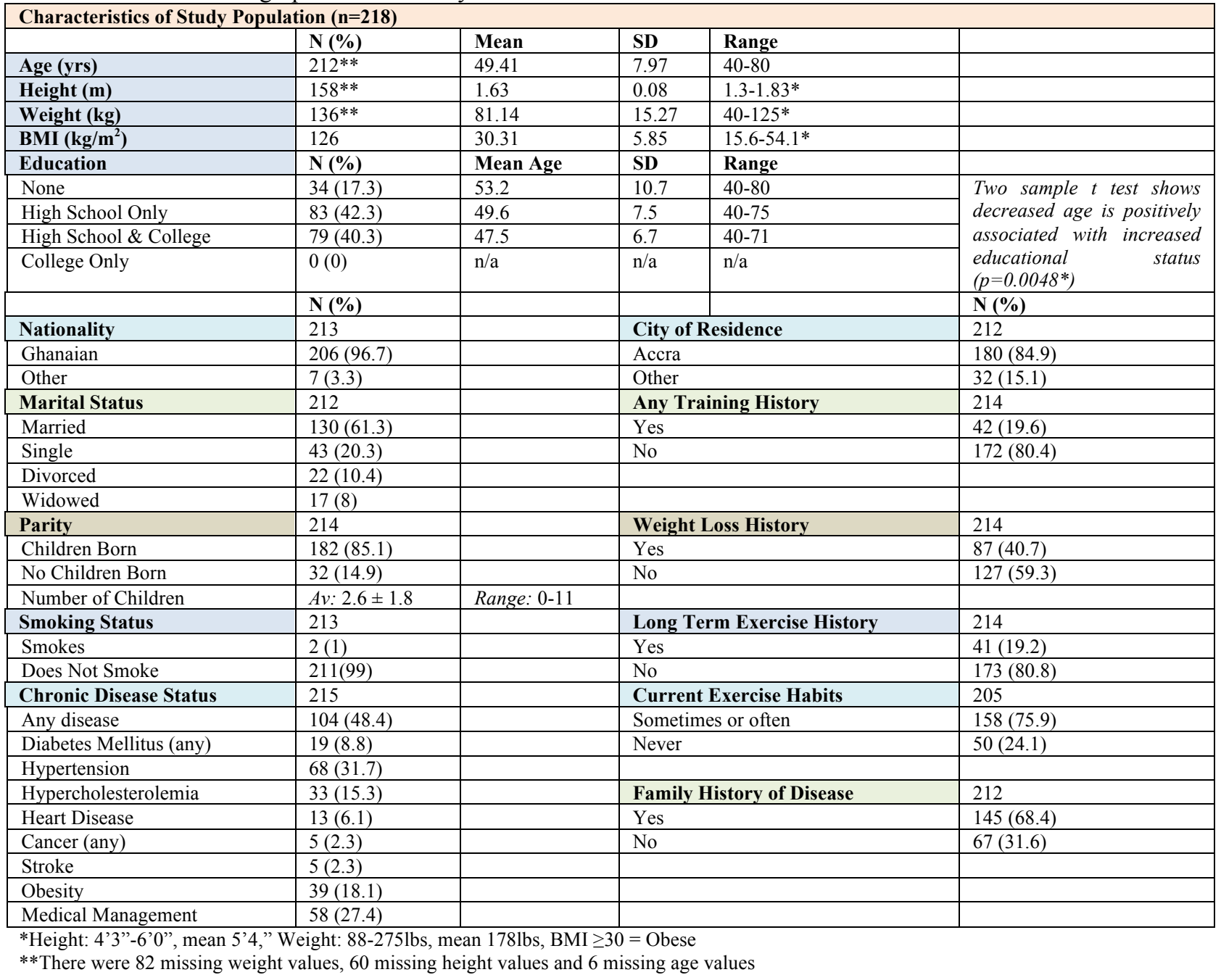


Specifically, 56.6\% $(\mathrm{n}=120)$ were between 40 and 49 , $33.0 \%(n=70)$ were between 50 and 59 , and $10.4 \%$ $(n=22)$ were above 60 . College education was negatively associated with age $(p=0.0048)$. The majority were married $(61.3 \% ; n=130)$ and had children $(85.1 \%$; $\mathrm{n}=182$ ). Number of live births ranged from 1 to 11 . There was no statistically significant relationship between age and the status "single" or "divorced". A higher percentage of younger women were married and when comparing older women to younger women, there was a statistically significant increase in the frequency of "widowed" status $(\mathrm{p}=<0.001)$.

\section{Overweight, obesity and smoking}

The mean BMI among the group was $30.3 \mathrm{~kg} / \mathrm{m}^{2}$. Consistent with previously reported data, married women had a statistically significant 3.5 unit increase in BMI when compared to unmarried women (adjusted for age, education and parity, $\mathrm{p}=0.002)$. ${ }^{6}$ Obesity was reported among $18 \%(n=39)$ of the cohort. The majority of the group $(99 \%, n=211)$ were reportedly non-smokers at the time of the survey.

Weight was inconsistently reported. To determine if weight missingness was non-random, weight missingness was regressed on twelve covariates. After adjusting for age, education, marital status, disease history, "weight loss" motivator, toning motivator, spouse motivator, beauty barrier, self-consciousness barrier, "no joy" barrier, no results barrier and hassle barrier, only education, positive disease status and the toning motivator were weakly negatively associated with weight non-reporting (AOR 0.9 for all; $\mathrm{p}=0.015,0.002$ and 0.047 , respectively).

No motivators or barriers were significantly associated with weight non-reporting. No systematic differences in age, marital status or parity distinguished weight reporters from weight non-reporters. Respondents without a college education were significantly more likely to be weight non-reporters $(\mathrm{p}=0.013)$. BMI was a calculated variable dividing self-reported weight in kilograms by self-reported height in meters squared. As self-reported weight was sensitive to non-response, BMI was not calculated for $42 \%$ of the sample.

Further analyses revealed that self-reported obesity was associated with a statistically significant 4.5 unit increase in BMI after adjusting for age, education, marital status and parity. Moreover, the specificity (95\%) of self-reporting obesity as a measure of calculated, clinical obesity was high.

Thus, to facilitate more complete use of the data, selfreported obesity was used instead of BMI in multiple analyses.

\section{$P A$ habits}

The majority of women $(75.9 \%, \mathrm{n}=158)$ reported exercising sometimes or often, $19.6 \%(n=42)$ reported having previously engaged in group- and/or personal training, and $24.1 \%(n=50)$ reported never having exercised. Over half of the women $(59.3 \%, n=127)$ denied ever successfully losing weight through PA. When controlling for age, education and marital status, there was a statistically significant association between education and history of personal and/or group training $(p=0.025)$, but not between age, education and marital status, and history of long-term exercise or exerciseinduced weight loss.

\section{Health status}

Personal history of chronic disease was reported among $48.4 \%(n=104)$, while $68.4 \%(n=145)$ expressed family history of chronic disease. The most commonly reported disease was hypertension, with a $31 \%$ cohortprevalence $(n=68)$. When compared to countrywidedata, hypertension was more common in our cohort than in the general population (31\% versus $24.8 \%$ countrywide). ${ }^{21}$ Reported prevalence of diabetes mellitus approximated countrywide prevalence $(8.8 \%$ vs. $6.3 \%$, respectively). ${ }^{8}$

Positive disease status was associated with a statistically significant 3.7 unit increase in BMI $(p=0.001)$ when compared to negative disease status (adjusted for age, education and marital status). ${ }^{8}$

\section{$P A$ preferences}

Low-intensity exercises such as walking, group classes at the gym and dancing were "favorites." Walking was most commonly reported $(69 \%, n=150)$.

\section{Motivators for and barriers to $P A$}

Women were instructed to select three primary PA motivators out of nine options (health/medical reasons, improve my mood, increase my energy levels and/or increase my self-confidence, reduce stress, lose weight, tone my muscles, spend time with friends, in order to please my spouse, I enjoy exercise, and other); and three primary barriers to PA, out of nine options (can't find the time to do it, family and/or work obligations, exercise is strenuous or painful, I do not enjoy exercise, I feel self-conscious when I exercise, exercise is a hassle, exercise makes managing my hair, hairstyle, skin or makeup a problem, I do not have a facility where I can exercise, I do not get results, other).

The top three motivators for PA were "Weight Loss," "Health Concerns" and "Increased Energy". The top three barriers to PA were "Can't Find the Time to Do It," "Work and Family Obligations," and "Don't have a Facility”. Figure 2 details these findings. 


\section{Analysis of top motivators \\ Weight loss motivator}

The correlates of the "weight loss" motivator were both internal (individual) and external (social) variables. Married women with a personal history of disease, or family history of disease or obesity were between 2 and 7 times more likely to select slimming as a motivator for PA $(p=<0.01-0.042)$. Women without a personal or familial history of disease or obesity had lower odds of selecting weight loss as a motivator. Women with a self-reported history of successful, exercise-induced weight loss were 3.36 times more likely to select weight loss as a motivator for exercise $(p=<0.001)$.

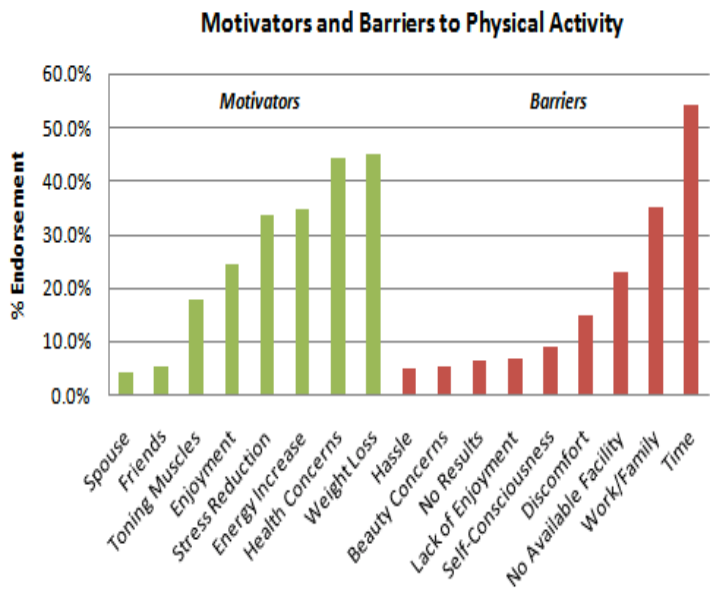

Figure 2 Prevalence of endorsement for all motivators and barriers to physical activity

\section{Health motivator}

Individual health and fitness status was the primary correlate of the "health" motivator. Women with a history of disease, exercise-induced weight loss or longterm exercise had approximately 2 times higher odds of endorsing health as motivation for exercise $(\mathrm{p}=0.041$, 0.017 and 0.055 respectively). Demographic data such as age, marital status, education and parity was not correlated with this motivator.

\section{Increased energy motivator}

No covariates were significantly correlated with the "increased energy" motivator.

\section{The relationship between common diseases and moti-} vators

Further regression analyses were performed to uncover possible correlations between the top three motivators (weight loss, health and increased energy) and presence of hypertension, hypercholesterolemia, diabetes, heart disease, cancer, stroke, or obesity. Both hypertension and hypercholesterolemia significantly correlated with the "weight loss" motivator (AOR 2.59, p=0.008 and AOR 3.56, $\mathrm{p}=0.012$, respectively).

\section{Internal correlation of top motivators}

Regression analyses to uncover correlations between the top three motivators indicated associations between weight loss and health concerns. Adjusting for age, education, marital status, and BMI, women who endorsed weight loss had 3.2 fold increased odds of endorsing health concerns and women who endorsed health concerns had a 3.4 fold increased odds of endorsing weight loss $(\mathrm{p}=0.006$ and $\mathrm{p}=0.004$, respective1y).

\section{Analysis of top barriers \\ Time barrier}

Women with a personal history of chronic disease (not including obesity) were $56 \%$ less likely to mention "time" as a barrier to PA (AOR 0.44, p=0.019). Women with any training history or long-term training history also had significantly decreased odds of endorsing the "time" barrier (AOR 0.44, $\mathrm{p}=0.038$ and AOR 0.35, $\mathrm{p}=0.008$, respectively). Parity was negatively yet insignificantly associated with the time barrier.

\section{Work and family obligations barrier}

Significant prevalence correlates of the "work/family obligations" barrier included successful exerciseinduced weight loss but not history of training or disease. Holding age, education, marital status and BMI constant, women with a history of exercise-induced weight loss had 2.48 greater odds of reporting work/family obligations as a barrier compared to those who did not report such weight-loss history $(p=0.006)$. There were no other covariates that were significantly associated with this barrier.

\section{Facility barrier}

Women who saw "no available facility" as a barrier to PA were $70 \%$ less likely to have a history of personal training when compared to women who did not report having no available training facility (unadjusted odds ratio $0.30, \mathrm{p}=0.032$, AOR $0.35, \mathrm{p}=0.063$ ).

Other

Outside of the available selections, six women reported "sickle cell anemia," "balance problems," "excess sweat," "I couldn't be bothered," "pure lack of focus" and "I feel lazy" as "other" barriers to PA. Five of the six respondents were married mothers with high school or college education. Average BMI among these respondents was $32 \mathrm{~kg} / \mathrm{m}^{2}$.

\section{Internal correlation of top barriers}

Adjusting for age, education, marital status and BMI, time was correlated with both work/family obligations (AOR 7.60, $\mathrm{p}=<0.001$ ) and facility limitations (AOR 5.56, $\mathrm{p}=<0.001)$. The facility barrier was associated with the time barrier (AOR 6.30, $\mathrm{p}=<0.001$ ). 
Work/family obligations and facility were not correlated.

\section{Projected PA profiles}

Correlations between motivators and barriers were further examined using scree plotting principal component and factor analysis in the STATA ${ }^{\text {TM }}$ software system. ${ }^{22}$
Seven factors with an Eigen value greater than one summarized age, all motivators, and all barriers. Of these seven, three factors loaded only single variables. These three were discarded and the four remaining summary factors were used to construct the respondent profiles summarized in Table 2 .

Table 2 Possible respondent profiles generated from a purposively selected cohort of women in urban Accra

\begin{tabular}{|l|l|l|l|}
\hline Factor & $\begin{array}{l}\text { Summary Statement (dom- } \\
\text { inant factor type) }\end{array}$ & $\begin{array}{l}\text { Highly Loaded Varia- } \\
\text { bles }\end{array}$ & Possible Respondent Profile \\
\hline $\mathbf{1}$ & $\begin{array}{l}\text { Healthy Lifestyle } \\
\text { (individual level factors) }\end{array}$ & $\begin{array}{l}\text { Motivators } \\
\text { Health } \\
\text { Energy } \\
\text { Weight loss } \\
\text { Barriers } \\
\text { Work } \\
\text { Time }\end{array}$ & $\begin{array}{l}\text { Age loads negatively on this factor. Busy, } \\
\text { working young women possibly interest- } \\
\text { ed in improving their health and fitness } \\
\text { but bound by work and family obliga- } \\
\text { tions }\end{array}$ \\
\hline $\mathbf{2}$ & $\begin{array}{l}\text { Socivators } \\
\text { Spending time witherm } \\
\text { friends } \\
\text { Barriers } \\
\text { Beauty concerns } \\
\text { Lack of a facility }\end{array}$ & $\begin{array}{l}\text { Age loads very weakly on this factor. } \\
\text { Midlife women possibly interested in the } \\
\text { social aspects of fitness who prize con- } \\
\text { venience and/or are service-oriented (i.e. } \\
\text { the culture of a fitness facility) }\end{array}$ \\
\hline $\mathbf{3}$ & $\begin{array}{l}\text { Motivators } \\
\text { Pleasing spouse } \\
\text { Barriers } \\
\text { Lack of results } \\
\text { Lack of enjoyment }\end{array}$ & $\begin{array}{l}\text { Age loads weakly on this factor. Midlife } \\
\text { women possibly frustrated by previous } \\
\text { experiences, cognizant of pressure from } \\
\text { spouse but without internal drive to do or } \\
\text { enjoy exercise }\end{array}$ \\
\hline $\mathbf{4}$ & $\begin{array}{l}\text { Motivators } \\
\text { Toning the muscles } \\
\text { Pure enjoyment }\end{array}$ & $\begin{array}{l}\text { Age loads highly on this factor. Retired, } \\
\text { older women who's maternal obligations } \\
\text { have thinned, leaving time to invest in } \\
\text { personal health }\end{array}$ \\
\hline
\end{tabular}

\section{DISCUSSION}

\section{Motivators and barriers}

The most frequently endorsed motivator was weight loss, which was correlated with marital status, personal history of disease, family history of disease and obesity. Consistent with countrywide data, marital status was positively associated with higher BMI $(p=0.002)$. The high frequency of endorsement suggests women associated PA with weight loss. Part of this may be explained by experience, as the correlation between history of exercise-related weight loss and endorsement of the weight loss motivator was significant.

The primary health motivator was significantly correlated with disease and fitness exposure. Our analysis suggests that women with a personal or family history of lifestyle-related disease, including obesity, saw weight loss as a means of health improvement and disease prevention.
This sub-population may have been informed that fitness promotes health via third party consultation (e.g. healthcare professional, fitness professional, fitness or personal partner). Energy was another top motivator for PA, but no variables were significantly correlated with it.

Consideration is given to the relatively non-descript but generally positive nature of the word "energy." Time was a top barrier to PA but women with personal disease exposure and training history were less likely to report this as a barrier. This may reflect women's experiences in time management aimed at incorporating health-related activities into daily life. Parity may not be associated with the time barrier because of the high prevalence of domestic helpers in Ghana.

Work/family obligations and poor access to facilities were also top barriers. Women who had a history of any PA training, but not long-term or weight-lossrelated training, had decreased odds of selecting lack of 
facility as a barrier. This disparity in odds may reflect varying conceptions of the terms "facility" and "training."

\section{Comparing Ghanaian priorities to other adults' prior- ities}

Data suggests that like their counterparts around the world, adult women in Ghana may be at risk of obesity and other lifestyle-related health detractors. Previously published studies address associations between perceptions (e.g., motivators and barriers) and PA among various groups of older adults. The Ghanaian cohort in this study reported exercise preferences, motivators and barriers that were both consistent and inconsistent with the findings from studies of age-matched populations outside Ghana. For example, in the U.S. and U.K., walking was also a reported as the preferred PA behavior among older adults. ${ }^{23}$ In a Korean-American study, health was also reportedly a key PA motivator. ${ }^{24}$ Moreover, as indicated by this study, research on American-Indians and African Americans noted time and space constraints as significant barriers to PA. ${ }^{14}$

However, unlike African-American women on whom PA data has been published, the Ghanaian women in our cohort did not weigh beauty concerns and social factors as primary motivators of PA. $5 \%(n=11)$ of the Ghanaian cohort selected "beauty concerns" as a barrier. This could reflect the relative infancy of formalized fitness programs or intentional PA among our cohort in urban Ghana, or the difference in socially acceptable female appearance(s) during and after exercise. Western women are exposed to fitness companies, apparel products and media campaigns that connect PA to aesthetic outcomes. In Ghana, no such fitness culture currently exists. Women's exposure to PA may be limited to contexts in which health (but not beauty) outcomes are promoted.

Recent data indicates that perceived ideal body weight among urban Ghanaian women is not likely to affect health-related interventions. ${ }^{3}$ Thus, despite unique perceptions of beauty including ideal body weight, consistent, mild-to-moderate PA programming is a feasible and simple way of stemming preventable chronic diseases among Ghanaian women. When their design incorporates cultural priorities, such programs can be tremendously effective. $^{13,25}$

\section{Limitations}

As a pilot study, this work was designed to collect formative data about a population that is largely ignored in the PA literature. As such, our analysis comprised inherent limitations. Since we sampled women from a single mega church, despite the diversity and largesse of the church, the data and conclusions are not generalizable to a larger population. A larger sample size and increased diversity in study site and data collection methodology are necessary in future large-scale studies.

Probing height and weight through self-reporting may have introduced misclassification error as height tends to be over-reported and weight is typically underreported in any study population. ${ }^{26}$ This misclassification error would affect BMI calculations. This limitation can be avoided by measuring height and weight on site. Variable interpretation of survey semantics may have occurred. Terms like "physical activity," "energy," "exercise," and "trainer" are interpreted differently depending on socio-cultural exposure and education. ${ }^{27}$ We tried to address this by including qualitative data in the analysis.

Coverage error may have been introduced. Women of limited mobility (older, disabled) may have been selfselected out as the sample convened in the church auditorium. Women interested in health, more readily perceiving value to the study, may have been overcovered. Furthermore, considering the study design and the relatively unique socio-economic status of our sample (e.g. high educational attainment), selection bias was introduced. The sample may have been biased towards women who were healthy and/or mobile and thus present at church, and towards women who were encouraged to pursue wellness through education. ${ }^{28,29}$

\section{CONCLUSION}

These data offer preliminary insights into the perceptions of PA, PA behaviors and health status in a specific cohort of Ghanaian women. To our knowledge, this population and similar groups have heretofore been ignored in inactivity and PA literature despite evidence that universally, PA can increase length and quality of life and reduce the risk of chronic disease ${ }^{30}$ and that age increases the risk of chronic disease while activity levels among many adult populations remain insufficient to produce positive health effects.

The data presented, in combination with emerging work on obesity and perceptions of health among various groups of West African women can direct interventions for disease prevention, health promotion, and fitness among this high-risk population.

\section{REFERENCE}

1. U.S. Department of Health and Human Services. Physical Activity and Health: A report of the Surgeon General. Atlanta GCfDCaP, National Center 
for Chronic Disease Prevention and Health Promotion; 1996

2. Frank LK, Heraclides A, Danquah I, Bedu-Addo $\mathrm{G}$, et al. Measures of general and central obesity and risk of type 2 diabetes in a Ghanaian population. Trop Med Int Health. 2012;18(2):141-51

3. Benkeser RM, Biritwum R, Hill AG. Prevalence of overweight and obesity and perception of healthy and desirable body size in urban, Ghanaian women. Ghana Med J. 2012;46(2):66-75

4. Abubakari AR, Lauder W, Jones MC, Kirk A, et al. Prevalence and time trends in diabetes and physical inactivity among adult West African populations: the epidemic has arrived. Public Health. 2009;123(9):602-14

5. World Health Organization. WHO global strategy on diet, physical activity and health: African regional consultation meeting report. Harare, Zimbabwe 18-20 March 2003

6. Abubakari AR, Lauder W, Agyemang C, Jones M, et al. Prevalence and time trends in obesity among adult West African populations: a meta-analysis. Obesity Reviews. 2008;9:297-311

7. Bhopal RS. Systematic review on the prevalence of diabetes, obesity/overweight and physical inactivity in Ghanaians and Nigerians. Public Health. 2008;122:173-182

8. Biritwum RB, Gyapong J, Mensah G. Epidemiology of Obesity in Ghana. Ghana Med J. 2005;39(3):82-85

9. Murison K, editor. Africa South of the Sahara 2004: Regional Surveys of the World. 33rd ed. London: Europa Publications; 2003

10. Eyler AA, Matson-Koffman D, Young DR, Wilcox S, et al. Quantitative study of correlates of physical activity in women from diverse racial/ethnic groups: The Women's Cardiovascular Health Network Project--summary and conclusions. Am J Prev Med. 2003;25(3 Suppl 1):93-103

11. D'Alonzo KT, Fiscetti N. Cultural beliefs and attitudes of Black and Hispanic college-age women toward exercise. $J$ Transcult Nurs. 2008;19(2):175-83

12. Estabrooka P, Lee RE, Gyurcsik NC. Resources for physical activity participation: does availability and accessibility differ by neighborhood socioeconomic status? Ann Behav Med. 2003;25(2):100-4

13. Belza B, Walwik J, Shiu-Thornton S, Schwartz S, et al. Older adult perspectives on physical activity and exercise: voices from multiple cultures. Prev Chronic Dis. 2004;1(4):1-12

14. Henderson K, Ainsworth B. A synthesis about perceptions of physical activity among older African American and America Indian women. $\mathrm{Am} J$ Public Health. 2003;93(2):313-17
15. Faber M, Kruger H. Dietary intake, perceptions regarding body weight, and attitudes toward weight control of normal weight, overweight, and obese black females in a rural village in South Africa. Ethn Dis. 2005;15(2):238-45

16. Myo Z, Dick J, Stein K. Perceptions of overweight African women about acceptable body size of women and children. Curationis. 1999;22(2):27-31

17. Amoah AG. Sociodemographic variations in obesity among Ghanaian adults. Public Health Nutr. 2003;6:751-7

18. Chiang KC. "It Is Our Exercise Family": experiences of ethnic older adults in a group-based exercise program. Prev Chronic Dis 2008;5(1):1-12

19. Hartford Institute for Religion Research, on-line reference available at: http://hirr.hartsem.edu/megachurch/database.html

20. Lincoln YS, Guba EG. (1985) Naturalistic Inquiry. , Beverly Hills:, CA; Sage Publications; 1985

21. Amoah AG. Hypertension in Ghana: a crosssectional community prevalence study in greater Accra. Ethn Dis. 2003;13(3):310-5

22. Principal component and factor analyses are most effective with large data sets. An "n" of approximately 200 is considered "fair" in this system. An " $n$ " of 100 is considered "poor," an " $n$ " of 500 is considered very good and an " $n$ " of1000 is considered excellent

23. Morris JN, Hardman AE. Walking to health. Sports Med. 1997;23(5):306-32

24. Lim KC, Waters CM, Froelicher ES, Kayser-Jones JS. Conceptualizing physical activity behavior of older Korean-Americans: an integration of Korean culture and social cognitive theory. Nurs Outlook. 2008;56:322-29

25. Yancey AK, McCarthy WJ, Harrison GG, Wong WK, et al. Challenges in improving fitness: results of a community-based, randomized, controlled lifestyle change intervention. J Womens Health. 2006;15(4):412-28

26. Nyholm M, Gullberg B, Merlo J, LundgvistPersson C, et al. The validity of obesity based on self-reported weight and height: implications for population studies. Obesity. 2007;15:197-208

27. Tudor-Locke C. In their own voices: definitions and interpretations of physical activity. Womens Health Issues. 2003;13:194-99

28. Ogwumike OO, Kaka B, Adegbemigun B, Abiona T. Health-related and socio-demographic correlates of physical activity level amongst urban menopausal women in Nigeria. Maturitas 2012;73(4):349-53

29. Bergman P, Grjibovski AM, Hagstromer M, Bauman M, Sjostrom M. Adherence to physical 
activity recommendations and the influence of socio-demographic correlates - a population-based cross-sectional study. BMC Public Health. 2008;8:367
30. Cress ME, Buchner DM, Prohaska T, Rimmer J, et $a l$. Best practices for physical activity programs and behavioral counseling in older adult populations. J Aging Phys Act. 2005;13(1):61-74 